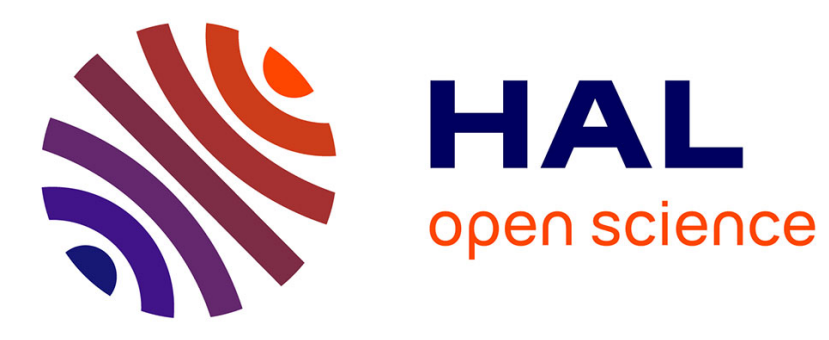

\title{
Pattern-based vision for microrobotic manipulators calibration and servoing
}

Valerian Guelpa, Patrick Sandoz, Cédric Clevy, Nadine Piat, Guillaume

Laurent

\section{- To cite this version:}

Valerian Guelpa, Patrick Sandoz, Cédric Clevy, Nadine Piat, Guillaume Laurent. Pattern-based vision for microrobotic manipulators calibration and servoing. International Conference on Ubiquitous Robots and Ambient Intelligence, Aug 2016, Xi'an, China. hal-03107814

\section{HAL Id: hal-03107814 https://hal.science/hal-03107814}

Submitted on 12 Jan 2021

HAL is a multi-disciplinary open access archive for the deposit and dissemination of scientific research documents, whether they are published or not. The documents may come from teaching and research institutions in France or abroad, or from public or private research centers.
L'archive ouverte pluridisciplinaire $\mathbf{H A L}$, est destinée au dépôt et à la diffusion de documents scientifiques de niveau recherche, publiés ou non, émanant des établissements d'enseignement et de recherche français ou étrangers, des laboratoires publics ou privés. 


\title{
Pattern-based vision for microrobotic manipulators calibration and servoing
}

\author{
Valérian Guelpa, Patrick Sandoz, Cédric Clévy, Nadine Le Fort-Piat, Guillaume J. Laurent \\ Automation and Micro-Mechatronics Systems Department, FEMTO-ST Institute \\ UMR CNRS 6174, ENSMM, Université de Franche-Comté, Besançon, France \\ (Tel : +33-3-81-40-28-08; E-mail: guillaume.laurent@ens2m.fr)
}

\begin{abstract}
This paper presents recent results obtained with a visual method allowing absolute position measurements at nanoscale. Based on the direct phase analysis of pseudo-periodic patterns, this approach enabled fast measurements of position with large range-to-resolution ratios. Applications include microrobotic manipulators calibration and visual servoing but also microforce measurement by using a compliant structure. Rangeto-resolution ratio of $10^{5}$ are typically overcome. For instance, the experiments have demonstrated a sub-nanometric resolution over a range of $168 \mu \mathrm{m}$, as well as a resolution below $50 \mathrm{nN}$ for a $50 \mathrm{mN}$ range for force measurement.
\end{abstract}

\section{INTRODUCTION}

In the last decade, high resolution visual methods based on periodic patterns has been proposed by different authors [1], [2]. For instance, in [2] sub-nanometer resolution is demonstrated in the measurement of 1D displacements of a compliant MEMS. In these methods, a periodic pattern is attached to a mobile object of interest. High resolution measurements are obtained from some Fourier-like processing which allows an easy calculation of the position directly from the phase value tied to the known grid's period. Nevertheless, the range of measurement is limited by the grid's period due the phase uncertainty of $2 \pi k$ given by the inverse tangent function implied in phase retrieval as shown in [2]. This means that the position measurement can be used to determine the target's position only within a single period.

To extend the range, embedded binary codes can be used as proposed in [3], [4], [5], [6]. These binary codes allow a coarse but absolute determination of the actual target's position allowing measurements larger than the grid's period and camera's FOV. The need to retrieve some binary code is however time-consuming and thus often incompatible with real-time applications.

Recently, we proposed a concept based on a twin-scale pattern with two different periods that allows a large range-toresolution ratio and a fast operating time [7], [8], [9]. In this paper, we presents some recent results on nanometric position and microforce measurement using this twin-scale method.

This paper is organized as follows. The measurement principle is presented in the next section. Section III and IV present respectively applications in micropositioning and microforce measurement.

\section{Twin-scale Visual Measurement Method}

The twin-scale method is based on direct phase measurement of two periodic patterns. The principle of the phase measurement method relies on the phase-to-displacement relationship that is a scalar product in the frequency domain:

$$
\mathcal{F}(f(x-\delta))=e^{-2 \pi i \delta \xi} \cdot \mathcal{F}(f(x))
$$

where $\mathcal{F}$ stands for the Fourier transformation. $f(x)$ is the space function considered (here the intensity of the pixels), $x$ the spatial coordinate, $\delta$ the spatial displacement and $\xi$ the reciprocal variable of $\mathrm{x}$ (or transform variable). A target displacement $\delta$ induces only a phase shift $\Phi$ in the frequency domain with $\Phi=2 \pi \delta \xi$.

Thanks to this linear relationship, we can retrieve object displacements with a very high precision through phase processing of the recorded images. In order to get efficient computation times, we compute a single-frequency spectral component instead of performing a complete Discrete Fourier Transform (DFT). To this end, a complex analysis vector $Z(k)$ defined by a Gaussian window and a periodic signal at the period $L_{p}$ (in pixels) of the stripe set is used:

$$
Z(k)=e^{-\left(\frac{k-N / 2}{N / 4.5}\right)^{2}} \cdot e^{-\left(\frac{2 i \pi(k-N / 2)}{L_{p}}\right)}
$$

where $k$ is the pixel index and $N$ is the image width in pixels.

The expected phase $\Phi$ is then given by the argument of the sum of each value of a transformed image vector, obtained by the dot product between the vector $Z$ and the vector of pixel intensities. The target displacement can then be determined by:

$$
\delta=\frac{\Phi \cdot L}{2 \pi}+n L
$$

where $n$ is an unknown integer standing for an entire number of stripe periods. Indeed due to the stripe periodicity, the displacement value is obtained modulo $L$ since different positions distant from an entire number of periods produce indistinguishable images.

To overcome this limitation, an efficient solution consists in using a twin-scale. A second stripe set with a slightly different period provides complementary and independent phase data that can be used for the removal of phase ambiguities. The principle is illustrated in Fig. 1. Thanks to the progressive mismatch between the two stripe sets, phase ambiguities can 
IIIII-

Twin stripe pattern

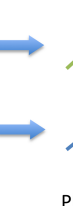

ase computations $\Rightarrow$ high resolution

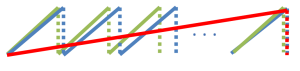

Removal of phase ambiguities $\Rightarrow$ extended range
Fig. 1. Working principle of the twin-scale visual measurement. See [8].

be removed and the unambiguous range switches from a single period to a new value $\Lambda$ given by:

$$
\Lambda=\frac{L_{1} \cdot L_{2}}{\left|L_{1}-L_{2}\right|}
$$

where $L_{1}$ and $L_{2}$ are the twin-stripe set periods.

The last step consists in using synthetic data to determine the correct $2 \pi m$ constant to apply to the phase shift observed for either stripe set:

$$
\delta=\frac{\Phi_{1} \cdot L_{1}}{2 \pi}+m L_{1}+p \Lambda
$$

in which $\Phi_{1}$ is the phase shift for the smallest stripe set, $m$ the number of periods derived from the synthetic phase and $p$ an unknown number of periods $\Lambda$ that represents the new ambiguity range.

Since $L_{1}$ and $L_{2}$ of the patterns are accurately known, the method is thus self-calibrated and does not depend on optical parameters such as magnification or field of view. Moreover the process of phase measurement is very robust to highfrequency spatial noises and exposure changes.

Photolithography processes were used to imprint the periodic pattern onto the substrate. The grid periods of $8 \mu \mathrm{m}$ and $8.4 \mu \mathrm{m}$ respectively were chosen that provide an absolute measurement range of $168 \mu \mathrm{m}$. This pattern is attached to the target of interest and is observed through a video microscope with low magnification lenses (typically $10 \times$ ) keeping a comfortable working distance in practice.

\section{Application to Nanoposition Measurements}

A series of experiments were performed in order to evaluate the method's capabilities in terms of repeatability, resolution and absolute positioning. The setup used in these experiments is presented in Figure 2. The vision system used in this work is composed of a red LED, a microscope objective , and a CCD camera $(780 \times 582$ pixels $)$ connected to a computer.

In order to evaluate the method's accuracy, a short range but high accurate PZT stage (P-753.1CD from Physics Instruments) was used. This stage has a resolution of $0.05 \mathrm{~nm}$ in closed-loop operation, and a repeatability of $1 \mathrm{~nm}$. The results of this experiment are depicted in Figure 3a. Figure 3b presents the difference between the measurement obtained from the capacitive sensor and with the twin-scale method. The standard deviation of this difference is $1.6 \mathrm{~nm}$, which is a good indicator of the method's accuracy [7]. Sub-nanometric resolution and $5 \mathrm{~nm}$ repeatability $(3 \sigma)$ were demonstrated in [8].

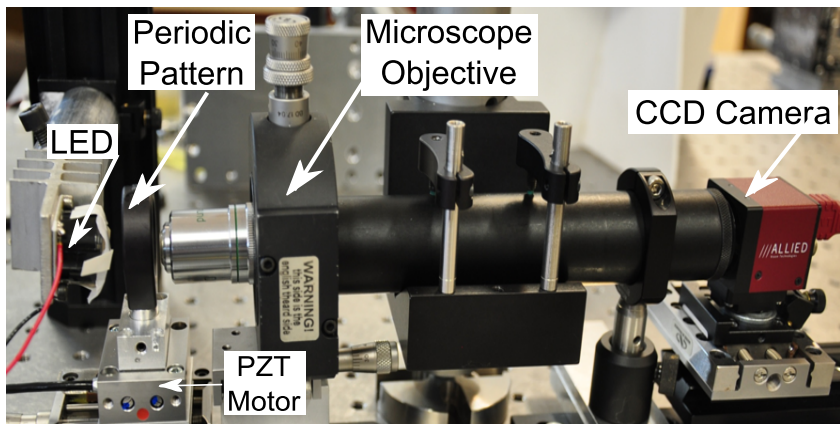

Fig. 2. Experimental set-up used in high-accurate position assessment.
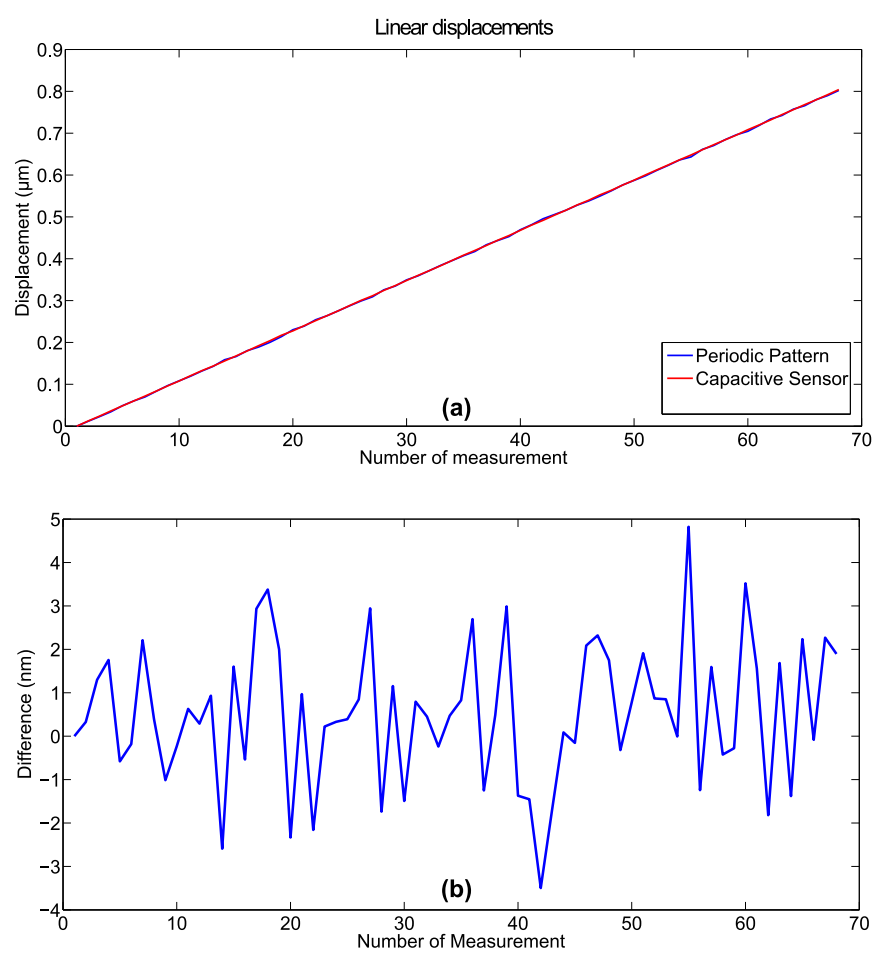

Fig. 3. Comparison of position values given by the PZT capacitive sensor and the twin-scale method: (a) positions returned by the capacitive sensor and by vision, (b) position differences.

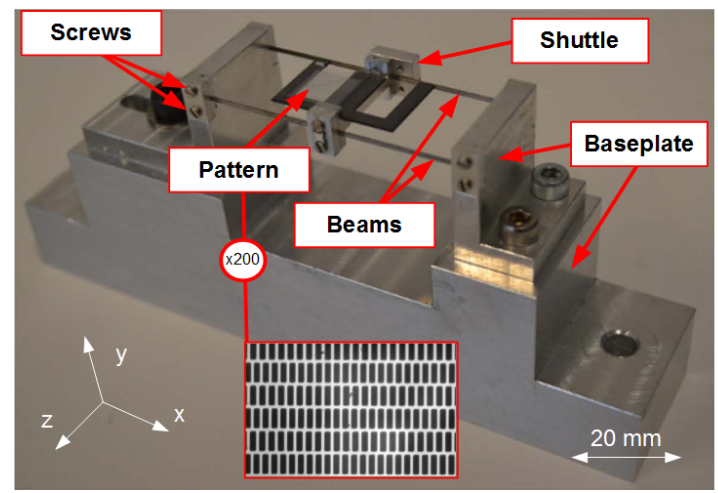

Fig. 4. The compliant structure for force measurement. The link between the shuttle and the base plate is four beams allowing the translation of the shuttle along the z-axis. 


\section{Application to Microforce Measurements}

The force measurement setup is based on a compliant structure presented in Fig. 4. It is composed of a mobile shuttle connected to its base plate by four thin compliant beams, restricting the motions along a single direction. We attached a twin-scale pattern on the shuttle in order to measure its position and displacements. From the knowledge of the stiffness between the shuttle and the base plate, we derive the forces applied to the shuttle from the measurement of its displacements. The stiffness is measured from the different frequencies of free oscillations with and without an additional known mass [9].

In order to determine the performances of the method, we realized measurements using a FemtoTools microforce sensing probe (model FT-S270) as reference sensor. This capacitive sensor has a force range of $2 \mathrm{mN}$ and a resolution of $0.4 \mu \mathrm{N}$. A second experimental setup has been used to quantify the full range of the sensor. In this case, the reference sensor was a calibrated precision balance (Mettler Toledo ML3002: repeatability $\sigma=0.01 \mathrm{~g} \simeq 100 \mu \mathrm{N}$; linearity $0.02 \mathrm{~g}$ $\simeq 200 \mu \mathrm{N})$.

The resulting data are plotted in Fig. 5 and shows good agreement and linearity between the visual and reference measurement tools. The repeatability of the measurement has been measured to $7.8 \mu \mathrm{N}$ and the resolution obtained experimentally is below $32.6 \mathrm{nN}$ [9].

\section{CONCLUSION}

In this paper, the twin-scale method has been successfully applied to nanometric position and micrometric force measurements. Position measurement is achieved with a sub-nanometric resolution and an unambiguous measurement range of $168 \mu \mathrm{m}$, leading to a range-to-resolution ratio in the order of magnitude of $10^{6}$. Similary, an experimental range of $50 \mathrm{mN}$ with a resolution below $50 \mathrm{nN}$ has been experimentally demonstrated for microforce sensing. Beyond these figured performances, the method shows also a flexibility welcomed at micro-scale: remote measurement, insensitivity to electronic noise, and camera self-calibration. These specifications make the method very attractive for a wide range of applications such as microrobotic manipulators calibration [10].

\section{ACKNOWLEDGEMENT}

This work has been supported by Région de BourgogneFranche-Comté, Labex ACTION project (ANR-11-LABX-0101) and Equipex ROBOTEX project (ANR-10-EQPX-44-01). Authors acknowledge the French RENATECH network and its FEMTO-ST technological facilities MIMENTO.

\section{REFERENCES}

[1] J. Gao, C. Picciotto, W. Wu, and W.M. Tong. From nanoscale displacement sensing and estimation to nanoscale alignment. Journal of Vacuum Science \& Technology B: Microelectronics and Nanometer Structures, 24:3094, 2006.

[2] C. Yamahata, E. Sarajlic, G.J.M. Krijnen, and M.A.M. Gijs. Subnanometer translation of microelectromechanical systems measured by discrete fourier analysis of ccd images. Microelectromechanical Systems, Journal of, 19(5):1273-1275, 2010.

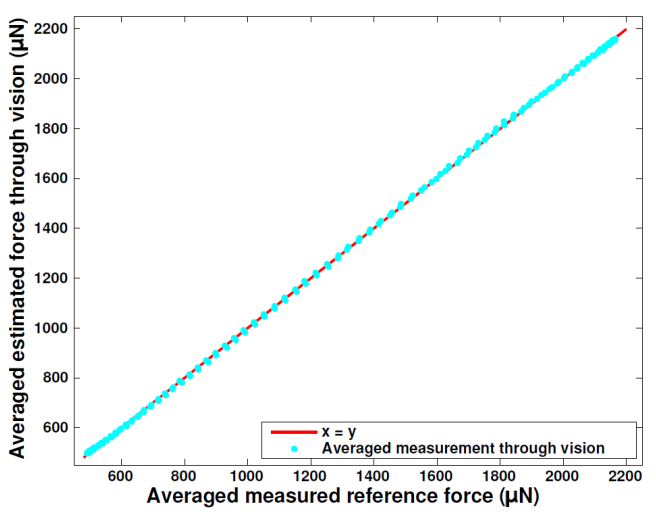

(a) Short range validation (average of 50 measurements for each point).

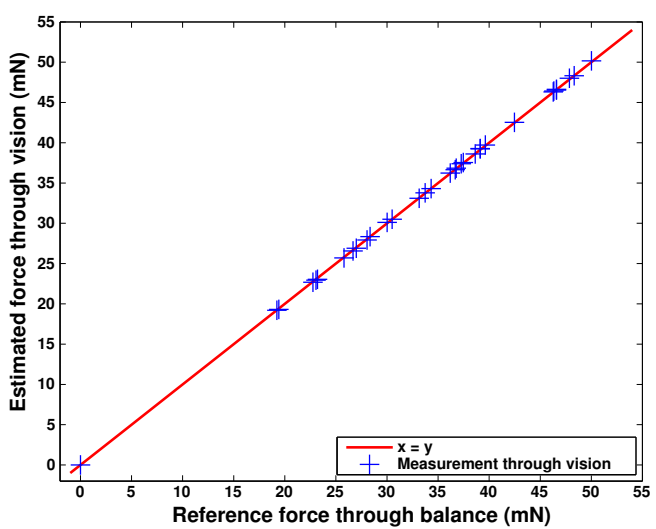

(b) Large range validation.

Fig. 5. Force measurements using the twin-scale method versus two different references.

[3] P. Masa, E. Franzi, and C. Urban. Nanometric resolution absolute position encoders. CSEM Scientific and Technical Report, pages 1-3, 2008.

[4] D.B. Boyton. Position encoder using statistically biased pseudorandom sequence, 2003. US Patent App. 10/399, 470.

[5] J. G. Zea, P. Sandoz, E. Gaiffe, J.L. Prétet, and C. Mougin. PseudoPeriodic Encryption of Extended 2-D Surfaces for High Accurate Recovery of any Random Zone by Vision. International Journal of Optomechatronics, 4(1):65-82, 2010.

[6] P. Sandoz, R. Zeggari, L. Froehly, J.L. Prétet, and C. Mougin. Position referencing in optical microscopy thanks to sample holders with out-offocus encoded patterns. Journal of Microscopy, 225(3):293-303, 2007.

[7] J. G. Zea, P. Sandoz, G. J. Laurent, L. L. Lemos, and C. Clevy. TwinScale Vernier Micro-Pattern for Visual Measurement of 1D In-Plane Absolute Displacements with Increased Range-to-Resolution Ratio . In International Symposium on Optomechatronic Technologies, volume 76, 2013.

[8] Valérian Guelpa, Guillaume J. Laurent, Patrick Sandoz, July Galeano Zea, and Cédric Clévy. Subpixelic measurement of large 1d displacements: Principle, processing algorithms, performances and software Sensors, 14(3):5056-5073, 2014.

[9] Valérian Guelpa, Guillaume J. Laurent, Patrick Sandoz, and Cédric Clévy. Vision-based microforce measurement with a large range-toresolution ratio using a twin-scale pattern. IEEE/ASME Transactions on Mechatronics, 20(6):3148-3156, 2015.

[10] N. Tan, C. Clévy, G. J. Laurent, P. Sandoz, and N. Chaillet. Accuracy quantification and improvement of serial micropositioning robots for inplane motions. IEEE Transactions on robotics, 31(6):1497-1507, Dec 2015. 